\title{
SELEÇÃO DE CATALISADORES E SISTEMA REACIONAL PARA A SÍNTESE DE ACETATO DE EUGENILA VIA CATÁLISE HETEROGÊNEA EM SISTEMA LIVRE DE SOLVENTE
}

\author{
D.A. LAROQUE ${ }^{1}$, R.A. $\operatorname{LOSS}^{1}$, M.J.A. SILVA ${ }^{1}$, J.V. OLIVEIRA ${ }^{1}$, D. OLIVEIRA ${ }^{1}$, H. \\ HENSE $^{1}$ \\ ${ }^{1}$ Universidade Federal de Santa Catarina, Departamento de Engenharia Química e \\ Engenharia de Alimentos \\ E-mail para contato: denise_laroque@ hotmail.com
}

\begin{abstract}
RESUMO - Acetato de eugenila é um éster que apresenta propriedades interessantes do ponto de vista industrial, porém, pequena disponibilidade na natureza. $\mathrm{O}$ presente trabalho objetivou avaliar diferentes sistemas reacionais e catalisadores na síntese do éster via catálise heterogênea a partir de óleo essencial de cravo-da-índia (85\% de eugenol) e anidrido acético, na razão molar 1:3, com $20 \%$ (m/m de substratos) dos catalisadores: peneira molecular $4 \AA$, resinas de troca iônica (Amberlyst 15, Amberlyst $15 \mathrm{H}^{+}$e Amberlyst 36) e resina não iônica (Amberlite XAD-16) em $2 \mathrm{~h}$ de reação a $55{ }^{\circ} \mathrm{C}$. Foram testados 3 sistemas reacionais: agitação com ultrassom e shaker e sem agitação. Todos os catalisadores testados foram eficazes para a síntese do éster, com conversões acima de $80 \%$, porém as reações com as resinas de troca iônica apresentaram coloração escura, necessitando de etapa subsequente de purificação. Desta forma, peneira molecular $4 \AA$ e resina não iônica Amberlite XAD-16, em sistema sem agitação, foram selecionados como catalisadores e sistema reacional para trabalhos futuros. Além disso, as reações estudadas empregaram condições reacionais simples e uso de tecnologia limpa, com potencial para aplicação em processos industriais.
\end{abstract}

\section{INTRODUÇÃO}

Os óleos essenciais são líquidos oleosos aromáticos obtidos a partir de material vegetal (flores, brotos, sementes, folhas, galhos, cascas, ervas, madeira, frutas e raízes) (Guenther, 1948). São conhecidos por suas propriedades aromáticas e medicinais, sendo largamente utilizados como conservantes de alimentos, antimicrobianos, analgésicos, sedativos, antiinflamatórios e anestésicos. Dentre os óleos essenciais, o de cravo-da-índia apresenta propriedades biológicas interessantes para aplicação na indústria de alimentos, farmacêutica e de cosméticos. Alguns autores atribuem as atividades benéficas ao seu composto majoritário, o eugenol, caracterizado pela baixa solubilidade e estabilidade (He et al., 2007; Campaniello et al., 2010; Devi et al., 2010). Porém, para contornar esses problemas, é possível a aplicação de metodologias de proteção de grupos, como por exemplo, através da acetilação por esterificação, produzindo assim, o éster acetato de eugenila, composto de pequena disponibilidade na natureza e com diversas propriedades de interesse industrial: atividade 
antioxidante (Kwang-Geun e Takayuki, 2001), atividade antimicrobiana (Chiaradia et al., 2012) atividade anticancerígena (Carrasco et al., 2008), inibe a descalcificação e promove a remineralização causada por erosão dentária (Charu et al., 2012). A reação para a obtenção do éster acetato de eugenila está ilustrada na Figura 1.

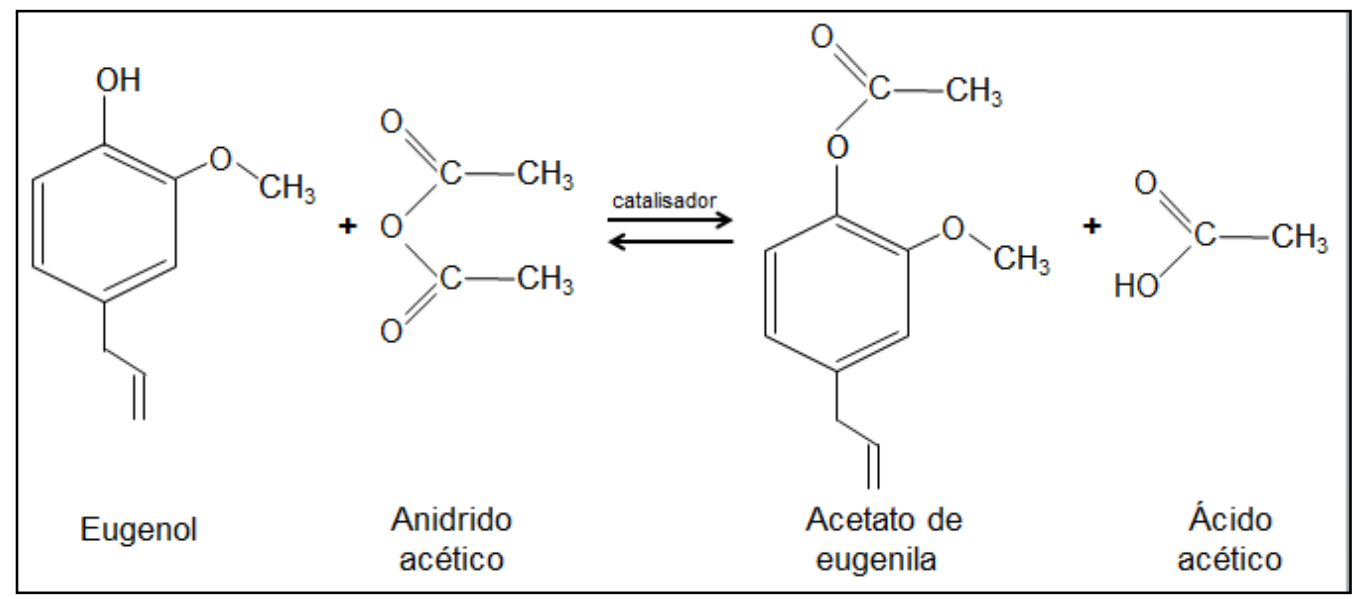

Figura 1 - Reação de síntese de acetato de eugenila. Fonte: Burdock (2010).

$\mathrm{O}$ acetato de eugenila foi avaliado pelo Joint FAO/WHO Expert Committee on Food Additives (JECFA) e pelo European Food Safety Authority (EFSA), sendo considerado seguro para o uso em alimentos e atualmente é listado no banco de dados da União Europeia de Substância Aromatizante, autorizado para utilização em alimentos (EFSA, 2011). A Tabela 2 apresenta os alimentos relatados com uso de acetato de eugenila (FEMA, 1994 apud BURDOCK, 2010). No Brasil, a lista de base ou de referência é a relação de todos os componentes aromatizantes com uso aprovado, no mínimo, por uma das entidades: JECFA, EU-CoE (European Union-Council of Europe), FDA (Food and Drug Administration) ou FEMA (Flavor and Extract Manufacturers Association) (ANVISA, 2007).

Segundo Sartori et al. (2004), extrema diversidade de catalisadores sólidos têm sido utilizados nas reações para adição de grupos protetores, sendo consequência à atenção dada ao meio ambiente, além da necessidade de melhoria nos métodos e de aumentar a seletividade dos grupos de proteção. A aplicação de catálise heterogênea na síntese orgânica simplifica a metodologia das reações, com atenção especial para a purificação do produto, possibilidade de reações com tempos curtos e condições moderadas. Pande e Samant (2013) estudaram a acetilação de compostos fenólicos e de álcoois utilizando anidrido acético e catalisadores heterogêneos. $\mathrm{O}$ catalisador polimérico Amberlyst 15 apresentou excelentes resultados para os vários compostos testados, chegando a obter $96 \%$ de conversão em 15 minutos de reação, além da possibilidade de reuso desses catalisadores, perdendo apenas $4 \%$ da sua atividade até o quarto ciclo. Em outro estudo, realizado por Manawwer et al. (2014), utilizando o catalisador heterogêneo $\mathrm{Ni} / \mathrm{SiO}_{2}$ para acetilação de compostos fenólicos e álcoois, sob condições suaves de temperatura, obtiveram ésteres aromáticos com conversões máximas de 80 e $100 \%$ de seletividade, e possível reutilização do catalisador por até 4 ciclos.

Nesse contexto, o presente trabalho visa à síntese de acetato de eugenila através da reação de esterificação por acetilação do eugenol presente no óleo essencial de cravo-da-índia, em sistema livre de solvente testando a eficácia de sistemas reacionais e de catalisadores 
sólidos: peneira molecular $4 \AA$, resinas de troca iônica (Amberlyst 15, Amberlyst $15 \mathrm{H}^{+} \mathrm{e}$ Amberlyst 36) e resina não iônica (Amberlite XAD-16).

\section{METODOLOGIA}

\subsection{Materiais}

Óleo essencial de folha de cravo-da-índia (Eugenia caryophyllus) adquirido da Ferquima Indústria e Comércio de Óleos Essenciais (São Paulo, Brazil), e anidrido acético (Vetec, 97\%) foram usados como substratos nas reações de acetilação. Os catalisadores sólidos testados foram: peneira molecular $4 \AA$ (Merck Milipore), resinas de troca iônica (Amberlyst 15, Amberlyst $15 \mathrm{H}^{+}$e Amberlyst 36) (Sigma-Aldrich) e resina não iônica (Amberlite XAD-16) (Sigma-Aldrich), esses catalisadores podem sem observados na Figura 2.
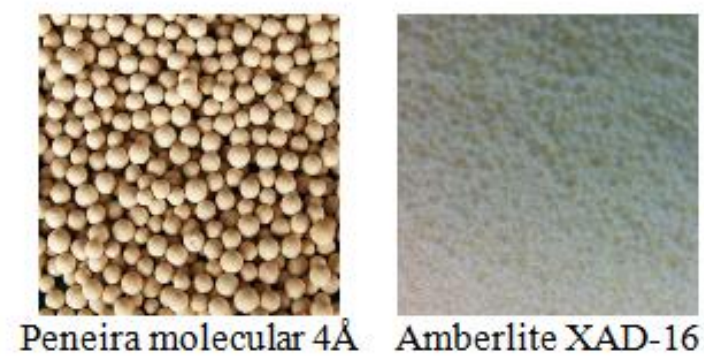

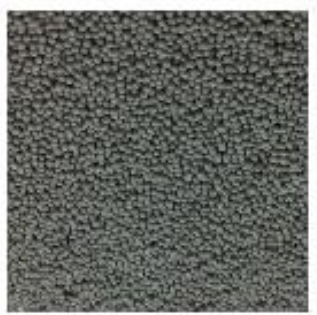

Amberlyst 36

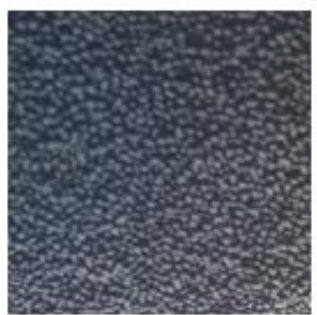

Amberlyst 15

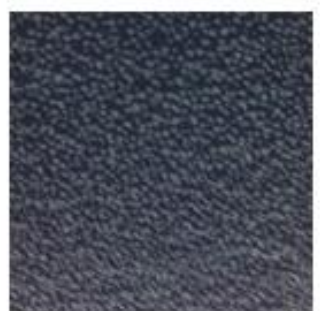

Amberlyst $15 \mathrm{H}^{+}$

Figura 2 - Catalisadores utilizados para síntese de acetato de eugenila.

\subsection{Reação de acetilação}

Os substratos óleo essencial de cravo-da-índia ( $85 \%$ de eugenol) e anidrido acético foram adicionados na razão molar de 1:3 (eugenol:anidrido acético) e após dissolução completa dos substratos, o catalisador sólido foi adicionado no teor de $20 \%$ e o tempo reacional de $2 \mathrm{~h}$ contado a partir da adição do mesmo, na temperatura de $55^{\circ} \mathrm{C}$. A peneira molecular $4 \AA$ foi testeda em 3 sistemas reacionais: com agitagão em Shaker (150 rpm) e ultrassom (100\% de potência, $132 \mathrm{~W})$ e sem agitação. E as resinas foram testadas apenas sem agitação. Após o término da reação, o catalisador foi filtrado em papel filtro e lavado com solvente (n-hexano). Após evaporação do solvente em rotaevaporador, as amostras foram mantidas sob refrigeração $\left(4^{\circ} \mathrm{C}\right)$ para posteriores análises.

\subsection{Determinação da conversão da reação}


A quantificação do éster produzido (acetato de eugenila) foi realizada por cromatografia gasosa (Shimadzu GC-2010). As análises foram realizadas utilizando coluna capilar de sílica fundida INOWAX (30 m x $250 \mu \mathrm{m}$ i.d.), $0,25 \mu \mathrm{m}$ de espessura de filme, detector FID, com a seguinte programação de temperatura: $40-180{ }^{\circ} \mathrm{C}\left(3{ }^{\circ} \mathrm{C} \min ^{-1}\right), 180-230{ }^{\circ} \mathrm{C}\left(20^{\circ} \mathrm{C} \min ^{-1}\right), 230$ ${ }^{\circ} \mathrm{C}$ (20 min), temperatura do injetor $250^{\circ} \mathrm{C}$, detector $275^{\circ} \mathrm{C}$, modo de injeção split, razão de split 1:100, gás de arraste $\mathrm{H}_{2}$, volume injetado $0,4 \mu \mathrm{L}$ de amostra diluída em diclorometano (1:10). A determinação da conversão das reações foi realizada acompanhando a redução na área do sinal do agente limitante (eugenol).

\section{RESULTADOS E DISCUSSÃO}

A Tabela 1 apresenta os resultados das conversões em acetato de eugenila para os catalisadores testados. Os cinco catalisadores sólidos conduziram a conversões elevadas, acima de $82 \%$ de acetato de eugenila. $\mathrm{O}$ aspecto visual de cada reação pode ser observado na Figura 3.

Tabela 1 - Conversões de acetato de eugenila via catálise heterogênea em diferentes sistemas reacionais.

\begin{tabular}{|c|c|c|}
\hline Catalisador & Sistema reacional & Conversão (\%) \\
\hline Peneira molecular $4 \AA$ & Ultrassom (100\%) & 84,8 \\
\hline Peneira molecular $4 \AA$ & Shaker (150 rpm) & 87,2 \\
\hline Peneira molecular $4 \AA$ & Sem agitação & 85,2 \\
\hline Amberlite XAD-16 & Sem agitação & 100 \\
\hline Amberlyst 15 & Sem agitação & 100 \\
\hline Amberlyst $15 \mathrm{H}^{+}$ & Sem agitação & 100 \\
\hline Amberlyst 36 & Sem agitação & 82,0 \\
\hline
\end{tabular}

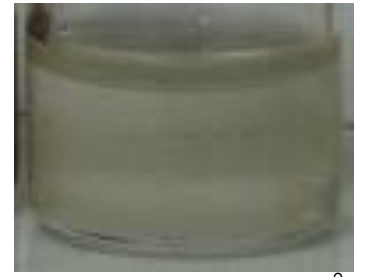

Peneira molecular $4 \AA$

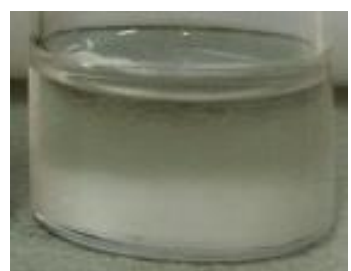

Amberlite XAD-16

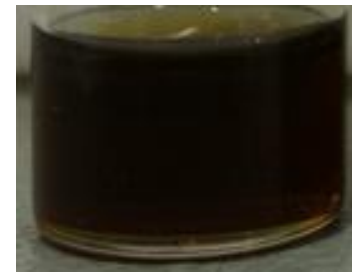

Amberyst 15

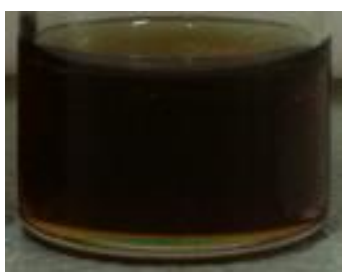

Amberlyst $15 \mathrm{H}^{+}$

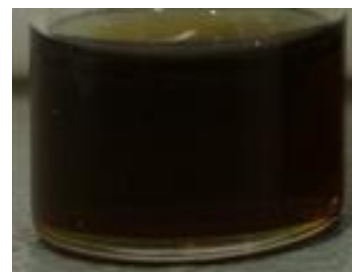

Amberlyst 36

Figura 3 - Aspecto visual das reações para a síntese de acetato de eugenila.

Para a peneira molecular $4 \AA$ as conversões nos três sistemas reacionais foram muito próximas (cerca de $85 \%$ ), a alta conversão no meio reacional sem agitação evidencia que as 
peneiras moleculares agem como "bombas moleculares", atraindo para o seu interior substratos pelos quais têm afinidade e após formação do produto, com características diferentes ao do substrato, expulsa-o para o meio reacional (Luna; Schuchardt, 2001). Porém, não foi encontrado nenhum estudo que utiliza peneiras moleculares como catalisadores em meio reacional sem agitação, esse resultado é de extrema importância, visto que pode diminuir o custo do processamento. Sá e Meier (2006) realizaram a acetilação de nucleosídeos empregando diferentes peneiras moleculares como catalisadores, os melhores resultados obtidos foram com as peneiras moleculares $13 \mathrm{X}$ e $4 \AA$ A, nas condições reacionais de $100{ }^{\circ} \mathrm{C}$ durante 6 horas de reação sob agitação.

Assim como para a peneira molecular, as resinas também apresentaram bons resultados em sistema reacional sem agitação, contudo, estudos encontrados na literatura utilizando resinas o sistema reacional é agitado em Shaker. Como estudos indicam que o efeito da limitação da difusão externa sobre a reação de esterificação está diretamente relacionada com a velocidade de agitação, Akyalçın e Altıokka (2012) estudaram esse efeito em diferentes velocidades (300, 500 e $700 \mathrm{rpm}$ ) em reação catalisada por Amberlyst 36, e observaram que a taxa de conversão é independente da velocidade de agitação, o que indica que a difusão externa, para a reação com essa resina, não é o controlada pela velocidade de agitação da reação.

As Amberlysts 15, $15 \mathrm{H}^{+}, 36$ apresentaram 100, 100 e 82\%, respectivamente, porém esses catalisadores de troca iônica, que apresentam grande potencial de esterificação de acetato de eugenila, são caracterizados pela coloração escura, alterando a cor da reação de amarelo claro para marrom escuro, logo, uma etapa adicional seria necessária para a remoção da coloração da reação, aumentando o tempo total e o custo do processo. Estudos que utilizam esses catalisadores para esterificação não relatam a mudança de coloração das reações (Liu et al., 2012; Yadav; Thathagar, 2002; Yadav; Kulkarni, 2000), porém, para a Amberlyst 40 wet (de coloração escura), em suas especificações o fabricante sugere que a coloração escura pode ser facilmente removida utilizando a Amberlyst 20-OH simultaneamente (ROHN and HASS Company, 2001).

A conversão em acetato de eugenila para a resina Amberlite XAD-16, de coloração amarelo claro, também foi de $100 \%$ sem agitação, evidenciando grande capacidade de esterificação. Na literatura não foi relatado o uso da Amberlite XAD-16 como catalisador em reações de síntese, o uso mais comum dessa resina é como adsorvente de compostos hidrofóbicos. Porém a Amberlyst 15 é amplamente estudada como agente catalisador em diversas reações. Lilja et al. (2002) empregaram catalise heterogênea para a esterificação de diferentes ácidos e os melhores resultados obtidos de tempo versus conversão foi para a resina Amberlyst 15. Sá et al. (2007) também utilizaram a resina Amberlyst 15 para a acetilação de $\alpha$-metileno- $\beta$-hidróxi ésteres e obtiveram conversões de $100 \%$ em 30 minutos de reação. No estudo da cinética para a síntese do éster laurato de citronelila via catálise enzimática, na presença do solvente heptano, a melhor conversão obtida foi de 53\% em 2 horas de reação, além da baixa conversão e do uso de solvente foi necessária a agitação do sistema reacional (Yadav; Lathi, 2004).

Chiaradia et al. (2012) também estudaram a síntese de acetato de eugenila, porém catalisada enzimaticamente. A conversão do eugenol foi realizada utilizando lipase comercial imobilizada de Candida antarctica como catalisador e anidrido acético como agente acilante. 
A melhor conversão foi de $99 \%$ usando razão molar eugenol:anidrido acético de 1:3, temperatura de $50{ }^{\circ} \mathrm{C}$ e teor de enzima $5,5 \%$, porém para obter esses resultados foram necessários 6 horas de reação e agitação em Shaker.

Os catalisadores testados nesse trabalho são uma alternativa atrativa para a produção do éster acetato de eugenila. A catálise heterogênea tem como vantagem a simplicidade na etapa de separação e purificação dos produtos da reação. É possível a utilização de menor quantidade de substratos e reações na ausência de solvente, além da possibilidade de reuso e regeneração, dessa forma, esses catalisadores, se enquadram no conceito de "química verde", com grande potencial para aplicação em processos industriais. Porém são necessários mais estudos para a determinação tempo reacional, quantidade de catalisadores e substratos. Através dos resultados obtidos os catalisadores peneira molecular $4 \AA$ e resina não iônica Amberlite XAD-16 e o sistema reacional sem agitação foram selecionados para trabalhos futuros.

\section{CONCLUSÃO}

Os catalisadores testados apresentaram grande potencial para a síntese de acetato de eugenila, sendo possível a obtenção de altas conversões em sistema reacional sem agitação. Porém nas reações com as resinas de troca iônica (Amberlyst 15, $15 \mathrm{H}^{+}$e 36 ) o meio reacional apresentou coloração escura, o que não ocorreu com a peneira molecular $4 \AA$ e a resina Amberlite XAD-16.

\section{REFERÊNCIAS}

AKYALÇIN, S.; ALTIOKKA, M.R. Kinetics of esterification of acetic acid with 1-octanol in the presence of Amberlyst 36. Appl.Catal. A: Gen., v. 429-430, p. 70-84, 2012.

BRASIL. Resolução RDC $n^{\circ}$ 2, de 15 de janeiro de 2007. Regulamento técnico sobre aditivos e aromatizantes. Disponível em <http:www.anvisa.gov.br/legis/resol/2007/rdc/02_170107rdc.htm>. Acesso em 29/01/2014.

BURDOCK, G.A. Fenaroli's handbook of flavor ingredients. $6^{\text {a }}$ ed., USA: Taylor and Francis Group, p. 681, 2010.

CAMPANIELlO, D.; CORBO, M. R.; SINIGAGLIA, M. Antifungal activity of eugenol against Penicillium, Aspergillus, and Fusarium species. J. Food. Prot., v. 73, p. 11241128, 2010.

CHARU, M.M.; SATIJA, G.; AVINASH, J.; NAGPAL, R.; KAPOOR, R.; AHMAD, A. In vitro inhibitory effect of clove essential oil and its two active principles on tooth decalcification by apple juice. Int. J. Dent., v. 2012, 2012.

CHIARADIA, V.; PAROUL, N.; CANSIAN, R.L.; JÚNIOR, C.V.; DETOFOL, M.R.; LERIN, L.A.; OLIVEIRA, J.V.; OLIVEIRA, D. Synthesis of eugenol esters by lipasecatalyzed reaction in solvent-free system. Appl. Biochem. Biotechnol., v. 168, nº. 4, p. $742,2012$. 
DEVI, K. P.; NISHA, S. A.; SAKTHIVEL, R.; PANDIAN, S. K. Eugenol (an essential oil of clove) acts as an antibacterial agent against Salmonella typhi by disrupting the cellular membrane. J. Ethnopharmacol., v. 130, p.107-115, 2010.

EFSA (European Food Safety Authority). Scientific Opinion on the safety and efficacy of allylhydroxybenzenes (chemical group 18) when used as flavourings for all animal species. EFSA Panel on Additives and Products or Substances used in Animal Feed (FEEDAP), EFSA Journal, Italy, v. 9, nº.12, 2011.

GUENTHER, E. The Essential Oils. New York: D. Van Nostrand, 1948.

HE, M.; DU, M.; FAN, M.; BIAN, Z. In vitro activity of eugenol against Candida albicans biofilms. Mycopathologia., v. 163, p.137-143, 2007.

KWANG-GEUN, L.; TAKAYUKI, S. Antioxidant property of aroma extract isolated from clove buds [Syzygium aromaticum (L.) Merr. et Perry]. Food Chem., v. 74, p. 443-448, 2001.

LILJA, J.; MURZINA, D.Y.; SALMIA, T.; AUMOA, J.; MÄKI-ARVELA, P.; SUNDELL, M. Esterification of different acids over heterogeneous and homogeneous catalysts and correlation with the Taft equation. J. Mol. Catal. A: Chem., v. 182-183, p. 555-563, 2002.

LIU, Z.; WANG, D.; WU, Y.; CHEN Y. Mild and efficient allylation of indoles and amides using Amberlyst-15 as a recyclable heterogeneous catalyst. Synth. Commun., v. 42, p. 1813-1823, 2012.

LUNA, F.J.; SCHUCHARDT U. Modificação de zeólitas para uso em catálise. Quím. Nova, v. 24, n . 6, p. 885-892, 2001.

MANAWWER, A.; ATEEQ, R.; NASER, M.A.; MOHAMMED, R.S. Ni/Silica catalyzed acetylation of phenols and naphthols: An eco-friendly approach. Arabian J. Chem., v. 7, p. 53-56, 2014.

PANDE, M.A.; SAMANT, S.D. Amberlyst-15 catalyzed acetylation of phenols and alcohols under solvent free conditions. Recyc. Cat., v.1, p. 13-16, 2013.

ROHN and HASS Company. Amberlyst 40 wet: For catalysis and separation Technologies. Comunicado técnico, 2001.

SARTORI, G.; BALLINI, R.; BIGI, F.; BOSICA, G.; MAGGI, R.; RIGHI, P. Protection (and deprotection) of functional groups in organic synthesis by heterogeneous catalysis. Chem. Rev., v. 104, p. 199-250, 2004.

SÁ M.M., MEIER L., FERNANDES L., PERGHER S.B.C. Acetylation of $\alpha$-methylene- $\beta$ hydroxy esters under heterogeneous catalysis: A regioselective preparation of MoritaBaylis-Hillman acetates. Catal. Commun., v. 8, nº 11, p. 1625-1629, 2007.

SÁ M.M., MEIER L. Pyridine-Free and Solvent-Free Acetylation of Nucleosides Promoted by Molecular Sieves. Accounts and Rapid Communications in Synthetic Organic Chemistry, n. 20, p. 3474-3478, 2006.

YADAV, G.D.; KULKARNI, H.B. Ion-exchange resin catalysis in the synthesis of isopropyl lactate. React. Funct. Polym., v. 44, p. 153-165, 2000. 
YADAV, G.D.; LATHI, P.S. Synthesis of citronellol laurate in organic media catalyzed by immobilized lipases: kinetic studies. J. Mol. Catal. B: Enzym., v. 27, p. 113-119, 2004.

YADAV, G.D.; THATHAGAR, M.B. Esterification of maleic acid with ethanol over cationexchange resin catalysts. React. Funct. Polym., v. 52, p. 99-110, 2002. 